\title{
Subtractive renormalization of chiral effective theory NN potentials up to next-to-next-to-leading order
}

\author{
C.-J. Yang*, Ch. Elster, and D. R. Phillips \\ Institute of Nuclear and Particle Physics, and Department of Physics and Astronomy, Ohio \\ University, \\ Athens, $\mathrm{OH}$ 45701, USA \\ E-mail: cjyang, elster, phillips@phy.ohiou.edu
}

We have developed a subtractive renormalization method with which we can evaluate nucleonnucleon $(\mathrm{NN})$ scattering phase shifts produced by the NN potential obtained at leading, nextto-leading, and next-to-next-to-leading order (NNLO) in chiral effective theory $(\chi \mathrm{ET})$. In this method the low-energy constants associated with short-distance NN physics are eliminated from the Lippmann-Schwinger equation (LSE) for the $\mathrm{NN}$ t-matrix, in favor of physical observables. This allows us to straightforwardly compute scattering phase shifts for ultra-violet cutoffs of at least $10 \mathrm{GeV}$. We then perform detailed analyses of the maximum cutoff at which the use of a $\chi$ ET NN potential in the LSE makes sense.

Specifically, we show that:

(a) our subtractive renormalization technique reproduces known results for the LO potential, in both S- and P-waves;

(b) a parameterization of short-distance physics in the NNLO potential in terms of an energydependent contact term creates scattering resonances and shallow bound states in S-wave channels once cutoffs larger than $1 \mathrm{GeV}$ are considered;

(c) the more conventional momentum-dependent contact term in the NNLO potential has problems of its own at cutoffs larger than $1 \mathrm{GeV}$;

(d) the NNLO potential yields P-wave phase shifts that have significant dependence on renormalization point.

(e) for cutoffs smaller than $1 \mathrm{GeV}$, using spectral-function regularization for the long-distance part of the potential produces results that vary with the cutoff and depend on the renormalization point less than if dimensional regularization is employed to compute the two-pion-exchange graphs.

Based on all these results we conclude that, once cutoffs larger than the chiral-symmetry breaking scale are employed, iteration of the two-pion-exchange piece of the $\chi$ ET NN potential in the LSE does not satisfy all of the criteria required for successful renormalization of the problem.

6th International Workshop on Chiral Dynamics, CD09

July 6-10, 2009

Bern, Switzerland

\footnotetext{
*Speaker.
} 


\section{Introduction}

Chiral perturbation theory $(\chi \mathrm{PT})$ is an effective field theory (EFT) that enables calculation in the non-perturbative region of QCD. The use of EFT in nuclear systems conveys two major advantages. First, we gain physical insight into the behavior of the strong interaction at large distances, which is difficult to calculate $a b$ initio from QCD. Second, our calculations become more accurate order by order, thus allowing systematic error control. An EFT becomes most powerful when a clear and large seperation between the low- and high-energy scale in the problem is possible. It has been shown that $\chi \mathrm{PT}$ is quite successful in the low-energy $(<1 \mathrm{GeV})$ mesonic sector. In principle, the same theory should work in the low-energy nucleon-nucleon (NN) sector as well.

However, it has been almost two decades since $\chi \mathrm{PT}$ was first applied to the problem of NN system[1], and difficulties still remain. Standard $\chi \mathrm{PT}$ power counting, which would predict no bound state for the deuteron, does not apply to the $\mathrm{NN}$ problem, because of infrared enhancements of the NN interaction. A nonperturbative treatment of at least part of the $\mathrm{NN}$ interaction is thus a necessary ingredient. One needs to either iterate the NN potential computed from $\chi \mathrm{PT}$ using the Lippmann-Schwinger equation (LSE) $[2,3,4,5,6,7,8,9,10,11,12,13,14,15,16,17,18,19]$, or determine which part of the potential can be treated in perturbation theory $[20,22,22,23,24$, $25,26,27]$. So far there is no consensus as to which of these two alternatives is superior.

Here, we adopt the former approach. In this "chiral effective theory" $(\chi \mathrm{ET})$ the behavior of the $\chi \mathrm{PT}$ potential at high momentum necessitates that a cutoff $\Lambda$ be placed on the momenta in the LSE. It is then natural to ask what values of $\Lambda$ can be used, if renormalization is to be successfully carried out. Difficulties in answering this question have both a technical part, i.e. it is hard to perform a fit for all unknown low energy constants at high cutoffs because of "fine-tuning"; and conceptual problems regarding what a successful renormalization is, e.g. is it sufficient that observables be (approximately) cutoff-independent? (See, e.g., Ref.[28], for a recent discussion.)

In Sec. 2 we outline a subtractive renormalization technique that solves the "fine-tuning" problem. This technique thus allows us to assess how well $\chi$ ET at large cutoffs satisfies criteria for successful renormalization. We have used subtractive renormalization to calculate the NN scattering amplitude obtained by using leading-order (LO), next-to-leading-order (NLO), and NNLO $\chi$ PT NN potentials in the LSE, for cutoffs up to $\Lambda=19 \mathrm{GeV}$. We show some results of these calculations in Sec. 3, and examine the conditions under which $\chi \mathrm{ET}$ is really improved, order by order, after renormalization. We do this for both the dimensionally-regularized (DR) and spectral-functionregularized (SFR) [29] $\chi \mathrm{PT}$ potentials, and consider both energy and momentum-dependent contact terms. More details regarding all these methods and results can be found in Refs. [30, 31, 32].

\section{Main ideas of subtractive renormalization}

The main idea of our subtraction method is to construct the fully off-shell partial-wave $t$-matrix from the knowledge of the long-range part of the potential and the on-shell value of the $t$-matrix for zero energy [30, 31, 32, 33, 34]. The partial-wave LSE is given by

$$
t_{l^{\prime} l}\left(p^{\prime}, p ; E\right)=v_{l^{\prime} l}\left(p^{\prime}, p\right)+\sum_{l^{\prime \prime}} \frac{2}{\pi} M \int_{0}^{\Lambda} \frac{d p^{\prime \prime} p^{\prime \prime 2} v_{l^{\prime} l^{\prime \prime}}\left(p^{\prime}, p^{\prime \prime}\right) t_{l^{\prime \prime} l}\left(p^{\prime \prime}, p ; E\right)}{p_{0}^{2}+i \varepsilon-p^{\prime \prime 2}} .
$$


Where $p_{0}^{2} / M=E$ is the center-of-momentum (c.m.) energy and $\Lambda$ the cutoff parameter. The incoming (outgoing) angular momenta are indicated by $l\left(l^{\prime}\right)$. The potentials are defined as:

$$
v_{l^{\prime} l}\left(p^{\prime}, p\right)=v_{l^{\prime} l}^{L R}\left(p^{\prime}, p\right)+C_{l^{\prime} l} p^{l^{\prime}} p^{l} f\left(p, p^{\prime} ; E\right),
$$

where $p\left(p^{\prime}\right)$ indicates the incoming (outgoing) momentum in the c.m. frame, $v_{l^{\prime} l}^{L R}$ is the long-range potential that is operative in this channel. $C_{l^{\prime} l} p^{\prime l^{\prime}} p^{l} f$ represents the contact interaction, where $f$ can be energy or momentum-dependent. First we consider the case $f=1$, which is the simplest contact term in a given partial wave. To relate the $t$-matrix to a physical quantity, a generalized scattering length for arbitrary angular momenta $l$ and $l^{\prime}$ can be defined as [35] $\frac{\alpha_{l^{\prime} l}}{M}=\lim _{k \rightarrow 0} \frac{t_{l^{\prime}}(k, k ; E)}{k^{l^{\prime}+l}}$, where for $l^{\prime}=l=0$ the usual definition, $\frac{\alpha_{00}}{M}=t_{00}(0,0 ; 0)$, is obtained. Dividing the partial-wave LSE, Eq. (2.1), by $p^{\prime l^{\prime}} p^{l}$ we obtain

$$
\frac{t_{l^{\prime} l}^{S J}\left(p^{\prime}, p ; E\right)}{p^{\prime l^{\prime}} p^{l}}=\frac{v_{l^{\prime} l}^{S J}\left(p^{\prime}, p\right)}{p^{\prime l^{\prime}} p^{l}}+\sum_{l^{\prime \prime}} \frac{2}{\pi} \frac{M}{p^{\prime l^{\prime}} p^{l}} \int_{0}^{\Lambda} \frac{d p^{\prime \prime} p^{\prime \prime 2} v_{l^{\prime} l^{\prime \prime}}^{S J}\left(p^{\prime}, p^{\prime \prime}\right) t_{l^{\prime \prime} l}^{S J}\left(p^{\prime \prime}, p ; E\right)}{p_{0}^{2}+i \varepsilon-p^{\prime \prime 2}} .
$$

Since $v_{l^{\prime} l}^{L R}\left(p^{\prime}, p\right) \sim p^{\prime l^{\prime}} p^{l}$, Eq. (2.3) is general and can be applied to any partial wave.

In the following we concentrate on P-waves $\left(l=l^{\prime}=1\right)$. (The corresponding argument for Swaves, in the case that we have the standard LO contact interaction of $\chi \mathrm{PT}$ with $l=l^{\prime}=0, f=1$, is analogous, but more straightforward, as division by a factor of $p^{\prime} k$ is not necessary there.) Consider the half-shell and on-shell $t$-matrices at $E=0$ :

$$
\begin{aligned}
\lim _{k \rightarrow 0}\left[\frac{t_{l^{\prime} l}\left(p^{\prime}, k ; 0\right)}{p^{\prime} k}\right] & =\lim _{k \rightarrow 0}\left[\frac{v_{l^{\prime} l}^{L R}\left(p^{\prime}, k\right)}{p^{\prime} k}+C_{l^{\prime} l}\right] \\
& +\sum_{l^{\prime \prime}} \frac{2}{\pi} M \lim _{k \rightarrow 0}\left[\frac{1}{p^{\prime} k} \int_{0}^{\Lambda} \frac{d p^{\prime \prime} p^{\prime \prime 2}\left(v_{l^{\prime} l^{\prime \prime}}^{L R}\left(p^{\prime}, p^{\prime \prime}\right)+C_{l^{\prime} l^{\prime \prime}} p^{\prime} p^{\prime \prime}\right) t_{l^{\prime \prime} l}\left(p^{\prime \prime}, 0 ; 0\right)}{-p^{\prime \prime 2}}\right] \\
\lim _{k \rightarrow 0}\left[\frac{t_{l^{\prime} l}(k, k ; 0)}{k k}\right] & =\lim _{k \rightarrow 0}\left[\frac{v_{l^{\prime} l}^{L R}(k, k)}{k k}+C_{l^{\prime} l}\right] \\
& +\sum_{l^{\prime \prime}} \frac{2}{\pi} M \lim _{k \rightarrow 0}\left[\frac{1}{k k} \int_{0}^{\Lambda} \frac{d p^{\prime \prime} p^{\prime \prime 2}\left(v_{l^{\prime} l^{\prime \prime}}^{L R}\left(k, p^{\prime \prime}\right)+C_{l^{\prime} l^{\prime \prime}} k p^{\prime \prime}\right) t_{l^{\prime \prime} l}\left(p^{\prime \prime}, 0 ; 0\right)}{-p^{\prime \prime 2}}\right]
\end{aligned}
$$

Subtracting Eq. (2.5) from Eq. (2.4) and multiplying both sides by $p^{\prime}$ cancels the unknown $C_{l^{\prime} l}$ :

$$
\begin{aligned}
\lim _{k \rightarrow 0}\left[\frac{t_{l^{\prime} l}\left(p^{\prime}, k ; 0\right)}{k}\right] & =\frac{\alpha_{11}}{M} p^{\prime}+\lim _{k \rightarrow 0}\left[\frac{v_{l^{\prime} l}^{L R}\left(p^{\prime}, k\right)}{k}\right]-p^{\prime} \lim _{k \rightarrow 0}\left[\frac{v_{l^{\prime} l}^{L R}(k, k)}{k^{2}}\right] \\
& -\sum_{l^{\prime \prime}} \frac{2}{\pi} M \int_{0}^{\Lambda} d p^{\prime \prime}\left[v_{l^{\prime} l^{\prime \prime}}^{L R}\left(p^{\prime}, p^{\prime \prime}\right)-\lim _{k \rightarrow 0}\left[\frac{v_{l^{\prime} l^{\prime \prime}}^{L R}\left(k, p^{\prime \prime}\right)}{k}\right] p^{\prime}\right] \lim _{k \rightarrow 0}\left[\frac{t_{l^{\prime \prime} l}\left(p^{\prime \prime}, k ; 0\right)}{k}\right](2.6)
\end{aligned}
$$

Here we have used that for P-waves $\lim _{k \rightarrow 0}\left[\frac{t_{11}(k, k ; 0)}{k k}\right]=\frac{\alpha_{11}}{M}$. The above limits are well-defined. The only unknown in Eq. (2.6) is $\lim _{k \rightarrow 0}\left[\frac{t_{l^{\prime} l}\left(p^{\prime}, k ; 0\right)}{k}\right]$, which can be solved by standard techniques.

The next step is to apply the same idea again to obtain $\frac{t_{l^{\prime} l}\left(p, p^{\prime} ; 0\right)}{p}$ and hence, $t_{l^{\prime} l}\left(p, p^{\prime} ; 0\right)$. We then proceed to calculate the on-shell $t$-matrix and the phase shifts using resolvent identities that connect the operator $t(E)$ to the operator $t(0)$. Those details are laid out in Refs. [34, 30, 38, 39].

Next, we consider an energy-dependent contact term in S-waves. We take $f C_{00}=\lambda+\gamma E$. This is the contact term up to NLO and NNLO in $\chi$ ET for the ${ }^{1} \mathrm{~S}_{0}$ channel. To simplify the presentation, 
we adopt the following operator notation for the LSE

$$
t(E)=\lambda+\gamma E+v_{L R}+\left[\lambda+\gamma E+v_{L R}\right] g_{0}(E) t(E),
$$

where $g_{0}(E)$ is the free resolvent of the LSE. Setting $E=0$ in Eq. (2.7) leads to $t(0)=\lambda+v_{L R}+$ $\left[\lambda+v_{L R}\right] g_{0}(0) t(0)$, which contains only one unknown, $\lambda$. Therefore, the matrix element $t\left(p^{\prime}, p ; 0\right)$ can be obtained from one experimental datum, here the $\mathrm{NN}$ scattering length $a_{0}$. After applying the same idea to obtain $t\left(E^{*}\right)$ from the phase shifts at an arbitrary energy, and using properties of the LSE to eliminate $\gamma$, we have

$$
\begin{aligned}
t(E)+t(0)\left[g_{0}(0)-g_{0}(E)\right] t(E)+ & \frac{E}{E^{*}}\left\{t(0)-\left[1+t(0) g_{0}(0)\right] \alpha t\left(E^{*}\right)\right\} g_{0}(E) t(E) \\
& =\left(1-\frac{E}{E^{*}}\right) t(0)+\frac{E}{E^{*}}\left[1+t(0) g_{0}(0)\right] \alpha t\left(E^{*}\right),
\end{aligned}
$$

where $\alpha \equiv\left[1+t\left(E^{*}\right) g_{0}\left(E^{*}\right)\right]^{-1}$. With $t(0)$ and $t\left(E^{*}\right)$ known, Eq. (2.8) is an equation for $t(E)$ and can be solved by standard methods with detail given in Ref. [32].

Finally, for (more complicated) contact terms such as

(A) $\lambda+C_{2}\left(p^{2}+p^{\prime 2}\right)$; (B) $\left(\begin{array}{cc}\lambda+C_{2}\left(p^{2}+p^{\prime 2}\right) & \lambda_{t} p^{\prime 2} \\ \lambda_{t} p^{2} & 0\end{array}\right)$; (C) $\left(\begin{array}{cc}\lambda+\gamma E & \lambda_{t} p^{\prime 2} \\ \lambda_{t} p^{2} & 0\end{array}\right)$,

we can solve the problem by combining the above methods, i.e., use the first subtraction to eliminate $\lambda$, and then relate $t\left(E^{*}\right)$ to $t(E)$. For coupled channels, we can apply the idea of dividing $p^{\prime l^{\prime}} p^{l}$ in LSE to eliminate $\lambda_{t} p^{2}$. However, in the case of the momentum-dependent S-wave contact terms, we need to perform one fitting to eliminate the unknown constant $C_{2}$. The inputs needed are:

for case (A); $a_{0}$, and an additional data to perform the fitting;

for case (B); $a_{0}, \alpha_{20}$, and an additional data to perform the fitting; and

for case $(\mathrm{C}) ; a_{0}, \alpha_{20}$ and phase shift at an arbitrary energy $\delta\left(E^{*}\right) .{ }^{1}$

\section{Results and discussion}

In this section we present our results in $\mathrm{P}$-waves and $\mathrm{S}$-waves to demonstrate the following:

(1). Our subtractive renormalization scheme generates results equivalent to the conventional "fitting" method, with a direct input of physical observables.

(2). The energy-dependent contact term produces phase shifts that oscillate with respect to $\Lambda$.

(3). Whether a contact term (or contact terms) is needed for a cutoff independent result is exactly determined by the (coordinate-space) singularity structure of the potential as $r \rightarrow 0$.

(4). Cutoff independence in the phase shift does not neccessarily mean the results are renormalizationpoint independent. Both properties are necessary conditions for successful renormalization.

(5). In general, there is a highest cutoff $\Lambda_{c} \approx 1$ (2) GeV in the LSE one can adopt for the NNLO DR (SFR) TPE, before the results start to exhibit problems.

To show point (1), we compare the LO S-wave phase shift obtained from the conventional "fitting" method to our subtractive scheme in the left-hand side of Fig. 1. Here the potential is the one-pion-exchange (OPE) plus a constant contact term. Fig. 1 shows that the results obtained

\footnotetext{
${ }^{1}$ The only restriction on $E^{*}$ is that it must be within the domain of validity of our theory.
} 

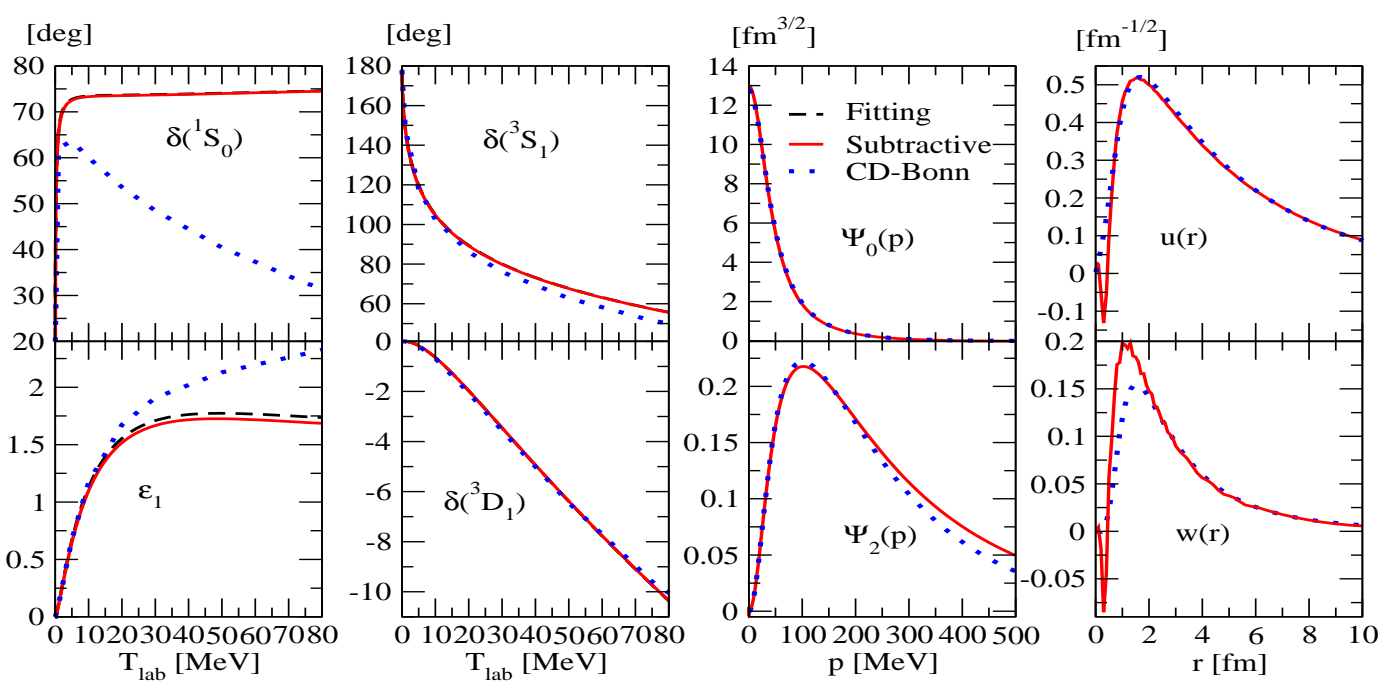

Figure 1: (Color online) The comparison of two renormalization methods for the lowest ${ }^{N N}{ }^{1} \mathrm{~S}_{0} \&{ }^{3} \mathrm{~S}_{1}{ }^{3} \mathrm{D}_{1}$ phase shifts (left two panels) and the bound-state wavefunctions (right two panels). $\psi_{0}(\mathrm{p})(\mathrm{u}(\mathrm{r}))$ is the ${ }^{3} \mathrm{~S}_{1}$ wavefunction and $\psi_{2}(\mathrm{p})(\mathrm{w}(\mathrm{r}))$ denotes the ${ }^{3} \mathrm{D}_{1}$ wave in momentum (coordinate) space. Here $\Lambda=50 \mathrm{GeV}$ is used. The dotted lines indicate the corresponding results obtained with the CD-Bonn potential [40].

by these two methods agree with each other within a relative difference of $2 \%$. (Due to numerical effects this difference is amplified in $\varepsilon_{1}$. $\varepsilon_{1}$ is well known for its sensitivity of the value of the unknown constant in the contact term, and we only adjusted $a_{0}$ up to certain precision when performing the "fitting" method.) We have verified that the off-shell t-matrices we obtain also agree with those obtained from the conventional "fitting" method to the same accuracy. Therefore, our subtraction method is as valid as the conventional fitting method. Our method can be applied to bound-state calculations too. The right-hand side of Fig. 1 shows deuteron wavefunctions obtained from our subtractive method, which are quite close to those obtained from the CD-Bonn potential.

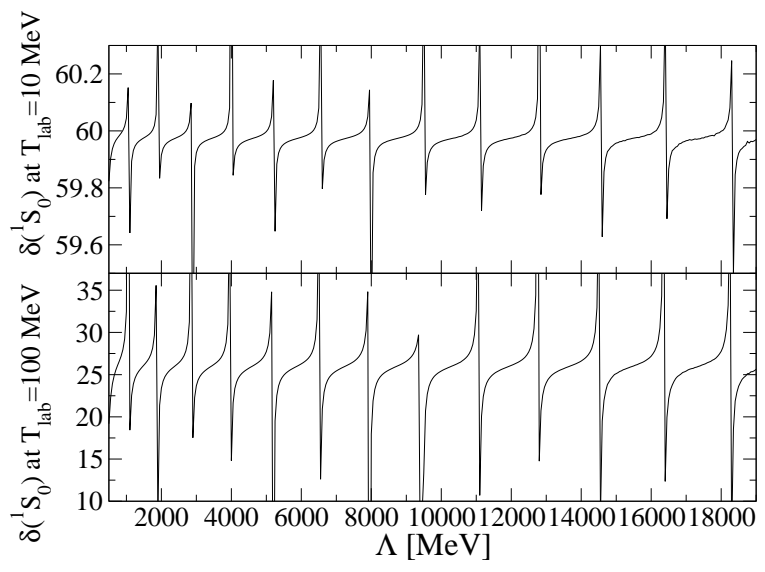

Figure 2: The ${ }^{1} \mathrm{~S}_{0} \mathrm{NN}$ phase shift at $T_{\text {lab }}=10$ (upper panel) and 100 (lower panel) $\mathrm{MeV}$ as a function of $\Lambda$. The results are obtained using the DR NNLO TPE with an energy-dependent contact term via our subtractive renormalization.

For point (2), we associate the DR NNLO TPE with the energy-dependent contact term and plot the ${ }^{1} S_{0}$ phase shifts versus $\Lambda$ in Fig. 2. The phase shifts show an oscillatory behvaior as a function of $\Lambda$. A similar oscillatory feature is observed in the ${ }^{3} \mathrm{~S}_{1}-{ }^{3} \mathrm{D}_{1}$ channel. (We use $a_{0}$, 


\begin{tabular}{|l|cccc|}
\hline & ${ }^{1} \mathrm{P}_{1}$ & ${ }^{3} \mathrm{P}_{0}$ & ${ }^{3} \mathrm{P}_{1}$ & ${ }^{3} \mathrm{P}_{2}$ \\
\hline \hline OPE & $\mathrm{U}$ & $\mathrm{R}$ & $\mathrm{U}$ & $\mathrm{R}$ \\
NLO (DR) & $\mathrm{U}$ & $\mathrm{U}$ & $\mathrm{R}$ & $\mathrm{R}$ \\
NNLO (DR) & $\mathrm{R}$ & $\mathrm{R}$ & $\mathrm{R}$ & $*$ \\
NLO (DR) + NNLO (SFR) & $\mathrm{U}$ & $\mathrm{U}$ & $\mathrm{R}$ & $\mathrm{R}$ \\
NNLO (SFR) & $\mathrm{U}$ & $\mathrm{U}$ & $\mathrm{R}$ & $\mathrm{R}$ \\
\hline \hline
\end{tabular}

Table I: Singularity structure of the long-range potentials $v_{11}^{L R}$. Here "U" ("R") means that $v_{11}^{L R}$ is repulsive (attractive) at $r \rightarrow 0$. The * indicates that both eigenpotentials in the ${ }^{3} \mathrm{P}_{2}-{ }^{3} \mathrm{~F}_{2}$ channel are attractive.
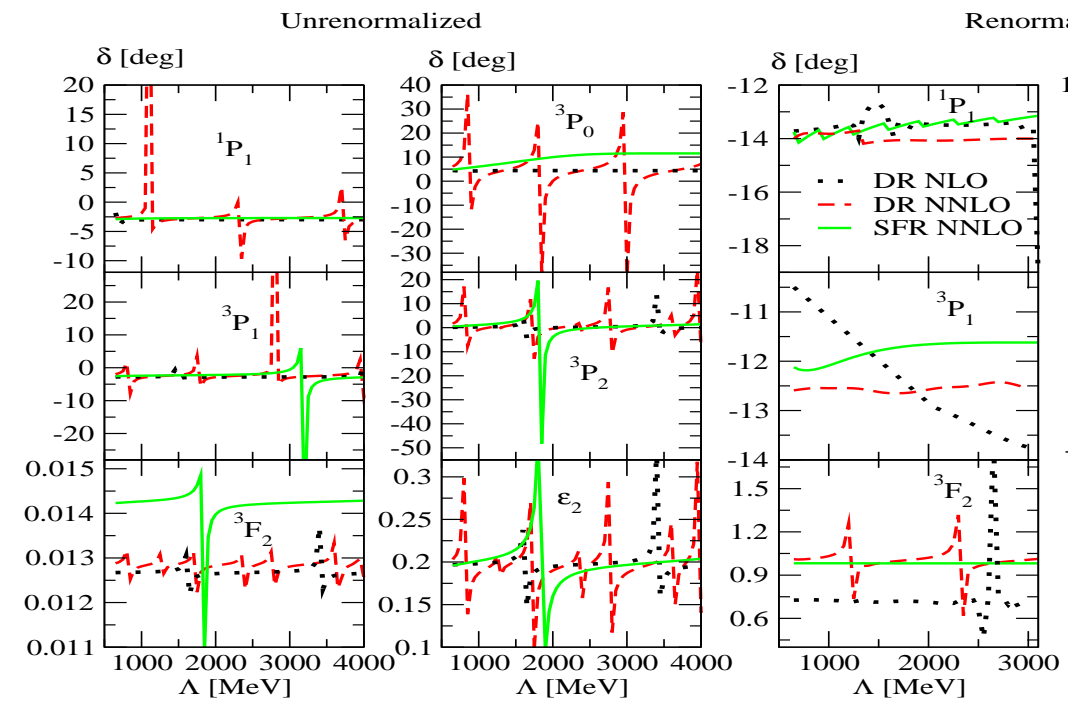

Renormalized

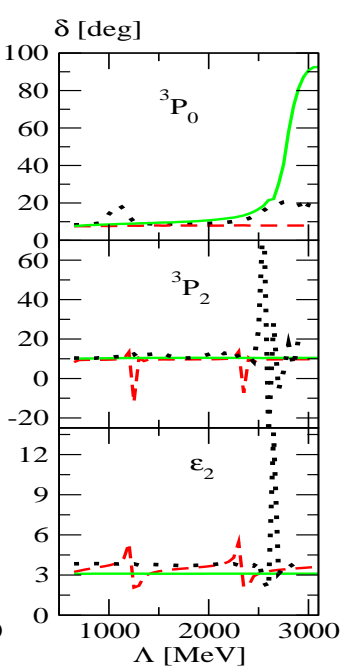

Figure 3: (Color online) The un-renormalized v.s. renormalized NN P-wave phase shifts at $T_{l a b}=10 \mathrm{MeV}$ (un-renormalized) and $100 \mathrm{MeV}$ (renormalized) as a function of $\Lambda$ for various $\chi$ PT potentials: DR NLO, black dotted line; DR NNLO, red dashed line; SFR NNLO, solid green line. For the renormalized case, the input $\alpha_{11}^{S J}$ were adjusted at each cutoff to give the best fit to the Nijmegen analysis [36] in the region $T_{l a b}<100 \mathrm{MeV}$.

$\delta\left(E^{*}\right)$ and $\alpha_{20}$ (for the triplet) as the input to generate the results with $E^{*}=1.4(10) \mathrm{MeV}$ for the singlet (triplet) channel.) This phenomenon is caused by the resonance state created by the energydependent potential. We emphasise that the first place where the phase shifts diverge is at cutoff $\Lambda \approx 1$ (1.2) GeV for the singlet (triplet) channel.

(3) involves the short distance $(r \rightarrow 0)$ behavior of $v_{l^{\prime} l}^{L R}$ in the coordinate space. We calculate the $r \rightarrow 0$ behavior analytically for various P-waves potentials and list them in Table I. At the same time, we plot the un-renormalized v.s. renormalized phase shifts at $T_{l a b}=10$ (100) MeV for TPE up to DR NLO, DR NNLO and the SFR TPE up to NNLO in Fig. 3. (Here, and throughout this paper, we adopt an intrinsic cutoff $\widetilde{\Lambda}=800 \mathrm{MeV}$ for the SFR TPE.) For the renormalized cases, the contact term has the form $C_{l^{\prime} l}^{S J} p^{\prime l^{\prime}} p^{l}$. Comparing the un-renormalized v.s. renormalized case indicates whether a contact term is needed for the phase shift to be stable with respect to $\Lambda$. This is exactly determined by the $r \rightarrow 0$ structure listed in Table I. If the potential is singular and attractive for $r \rightarrow 0$ (denoted as" $\mathrm{R}$ " in Table I), then the contact term is required. If it is not (" $\mathrm{U}$ " in Table I) then the phase shifts will have a stable $\Lambda \rightarrow \infty$ limit even in the absence of a contact term (see also Refs. [17, 18, 37]). The ${ }^{3} \mathrm{P}_{2}-{ }^{3} \mathrm{~F}_{2}$ channel for DR NNLO is a special case, since 
the coupled-channels potential has two attractive singular eigenpotentials in the $r \rightarrow 0$ limit, and so one subtraction is not sufficient to make phase shifts independent of $\Lambda$ in this channel.
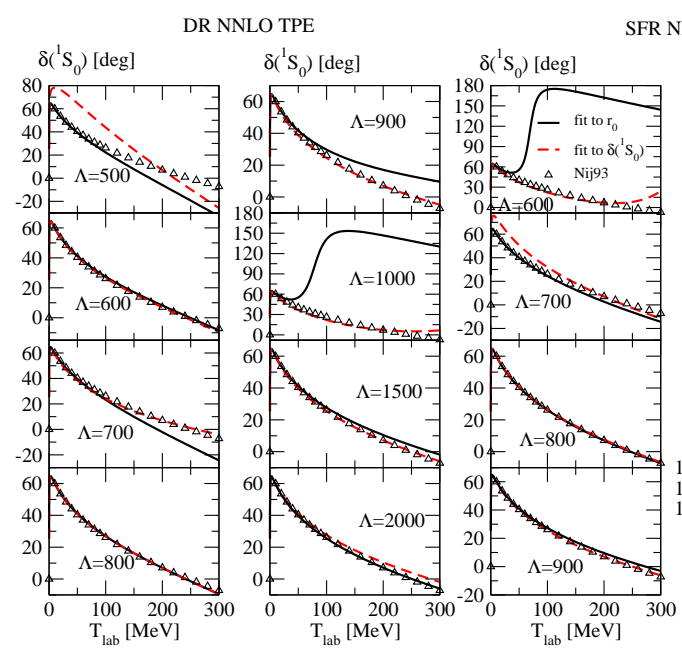

FR NNLO TPE

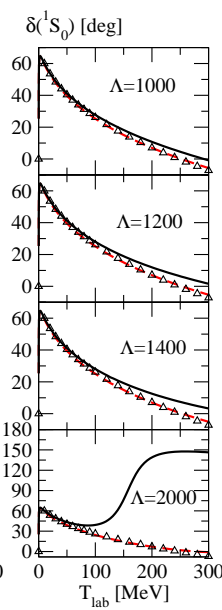

Figure 4: (Color online) The ${ }^{1} \mathrm{~S}_{0} \mathrm{NN}$ phase shift as a function of the lab. kinetic energy for various $\Lambda$. The results are obtained with the DR (left two panels) or SFR TPE (right two panels) up to NNLO with momentum-dependent contact terms. We use $a_{0}=-23.7 \mathrm{fm}$ as input and then perform a fit to either the effective range $r_{0}=2.7 \mathrm{fm}$ (solid black line) or the phase shift at $T_{l a b}=200 \mathrm{MeV}$ (dashed red line). The phase shifts [36] are denoted by open triangles.

(4) To see why a cutoff-independent result in the phase shift is not neccessarily renormalizationpoint-independent, we plot the ${ }^{1} S_{0}$ phase shift obtained with the DR and SFR NNLO TPE along with the momentum-dependent contact term (denoted as case (A) at the end of the previous section) in Fig. 4. As mentioned before, in this case we perform the renormalization by the one-subtractionplus-one-fitting procedure. The results obtained by fitting to the effective range $r_{o}$ or to the phase shift at $T_{l a b}=200 \mathrm{MeV}$ are shown. One can see that the two different fit procedures generate different results for the same $\Lambda$. This is especially visible at $\Lambda=500$ and $1000 \mathrm{MeV}$ for the DR NNLO TPE, where a resonance-like behavior is present in the latter case when $C_{2}$ is fitted to $r_{0}$. For values of $\Lambda$ not close to these problematic cutoffs the phase shift is almost independent of the renormalization point. In contrast, for the SFR TPE, the two different fitting procedures lead to almost the same phase shift for $\Lambda$ between $700-1800 \mathrm{MeV}$. By switching to the SFR TPE, we achieve renormalization-point-independence for a wider range of $\Lambda$.
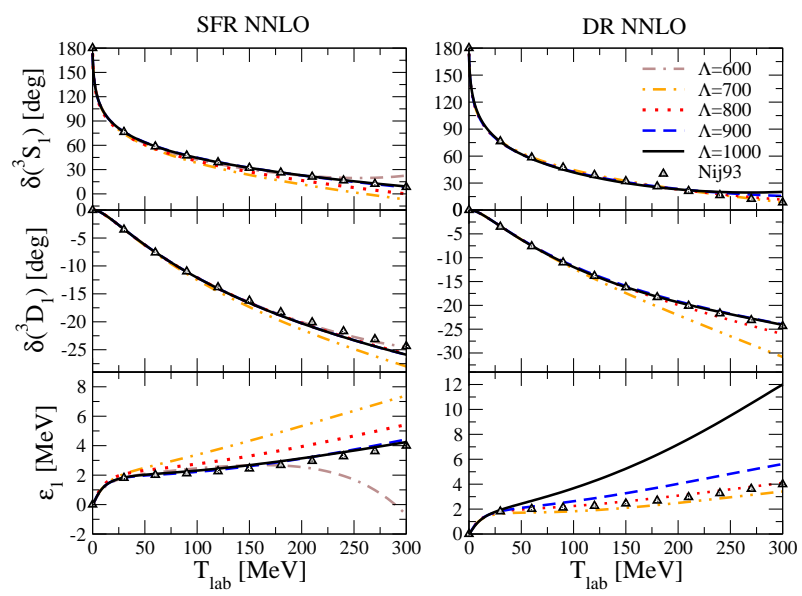

Figure 5: (Color online) The best fit for the $\mathrm{NN}^{3} \mathrm{~S}_{1}-{ }^{3} \mathrm{D}_{1}$ phase shifts as a function of the laboratory kinetic energy for different cutoffs $\Lambda$ ranging from 0.6 to $1 \mathrm{GeV}$. The potentials employed are the SFR NNLO with a momentum-dependent central part of the contact term. The values of the Nijmegen phase-shifts [36] are indicated by the open triangles.

Finally, for point (5), we plot the ${ }^{3} S_{1}-{ }^{3} D_{1}$ phase shifts in Fig. 5. These results are obtained by the SFR or DR TPE up to NNLO plus the momentum-dependent contact term (labeled as (C) 
in the previous section). For the DR TPE case, the best overall fit already diverges away from the Nijmegen analysis in the mixing angle at $\Lambda=1000 \mathrm{MeV}$. This implies that there is a critical cutoff $\Lambda_{c} \sim 1 \mathrm{GeV}$. Above that we cannot iterate DR NNLO TPE in LSE and obtaine a good fit in the ${ }^{3} S_{1}-{ }^{3} D_{1}$ channel. Moreover, as shown in Fig. 4, at this $\Lambda_{c}$ the renormalization-pointindependence also breaks down for the DR NNLO TPE in the ${ }^{1} \mathrm{~S}_{0}$ channel. Therefore, we conclude that for the DR TPE in S-waves, the highest cutoff one can adopt in the LSE is $\Lambda_{c} \sim 1 \mathrm{GeV}$. For the P-waves, a detail analysis of the renormalization-point-dependence suggests that $\Lambda_{c} \sim 1-1.2$ $\mathrm{GeV}$ for the DR NNLO TPE[31]. As with the S-waves, if the SFR TPE is adopted, then $\Lambda_{c}$ can be extented to $2 \mathrm{GeV}$ before similar problems appear.

\section{Summary and Conclusions}

We developed a subtractive renormalization scheme for $\chi$ ET NN potentials which allows us to go to an arbitrarily high cutoff in the LSE. Our calculations show that the energy-dependent contact term creates scattering resonances and shallow bound states in S-wave channels once cutoffs larger than $1 \mathrm{GeV}$ are considered. Momentum-dependent contact terms in the NNLO potential also has problems at these cutoffs. We also investigate the singularity structure of the potential and find that the LO conclusion presented in Ref.[15] holds up to NNLO. Our analysis in S-waves and P-waves shows that the two-pion-exchange potential should not be inserted in the Lippmann-Schwinger equation and treated non-perturbatively if cutoffs larger than $1 \mathrm{GeV}$ are employed.

\section{Acknowledgments}

This work was performed in part under the auspices of the U. S. Department of Energy, Office of Nuclear Physics, under contract No. DE-FG02-93ER40756 with Ohio University. We thank the Ohio Supercomputer Center (OSC) for the use of their facilities under grant PHS206.

\section{References}

[1] S. Weinberg, Phys. Lett. B 251, 288 (1990); Nucl. Phys. B 363, 3 (1991).

[2] C. Ordonez, L. Ray and U. van Kolck, Phys. Rev. C 53, 2086 (1996).

[3] N. Kaiser, R. Brockmann and W. Weise, Nucl. Phys. A 625, 758 (1997).

[4] G. P. Lepage, arXiv:nucl-th/9706029.

[5] E. Epelbaum, W. Glöckle, and U.-G. Meißner, Nucl. Phys. A 671, 295 (2000).

[6] J. Gegelia, Phys. Lett. B 463, 133 (1999).

[7] D. Eiras and J. Soto, Eur. Phys. J. A 17, 89 (2003).

[8] D. R. Entem and R. Machleidt Phys. Lett. B 524, 93 2002; Phys. Rev. C 68, 041001(R) (2003).

[9] J. A. Oller, Nucl. Phys. A 725, 85 (2003).

[10] E. Epelbaum, W. Glockle and Ulf-G. Meißner, Nucl. Phys. A 747, 362 (2005).

[11] D. Djukanovic, S. Scherer, M. R. Schindler and J. Gegelia, Few Body Syst. 41, 141 (2007). 
[12] S. R. Beane, P. F. Bedaque, M. J. Savage, and U. van Kolck, Nucl. Phys. A 700, 377 (2002).

[13] M. Pavón Valderrama and E. Ruiz Arriola, Phys. Lett. B 580, 149 (2004).

[14] M. P. Valderrama and E. R. Arriola, Phys. Rev. C 70, 044006 (2004).

[15] A. Nogga, R.G.E. Timmermans and U. van Kolck, Phys. Rev. C 72, 054006 (2005).

[16] E. Epelbaum and Ulf-G. Meißner, arXiv:nucl-th/0609037.

[17] M. Pavón Valderrama and E. Ruiz Arriola, Phys. Rev. C 74, 054001 (2006).

[18] M. P. Valderrama and E. R. Arriola, Phys. Rev. C 74, 064004 (2006) [Erratum: C 75, 059905 (2007)].

[19] D. R. Entem, E. Ruiz Arriola, M. P. Valderrama and R. Machleidt, Phys. Rev. C 77, 044006 (2008).

[20] D. B. Kaplan, M. J. Savage and M. B. Wise, Nucl. Phys. B 478, 629 (1996); Phys. Lett. B 424, 390 (1998); Nucl. Phys. B 534, 329 (1998).

[21] S. Fleming, T. Mehen and I. W. Stewart, Nucl. Phys. A 677, 313 (2000).

[22] S. Fleming, T. Mehen and I. W. Stewart, Phys. Rev. C 61, 044005 (2000)

[23] S. R. Beane, D. B. Kaplan and A. Vuorinen, arXiv:0812.3938 [nucl-th].

[24] M. C. Birse and J. A. McGovern, Phys. Rev. C 70, 054002 (2004).

[25] T. Barford and M. C. Birse, Phys. Rev. C 67, 064006 (2003).

[26] M. C. Birse, Phys. Rev. C 74, 014003 (2006).

[27] B. Long and U. van Kolck, Annals Phys. 323, 1304 (2008).

[28] E. Epelbaum and J. Gegelia, arXiv:0906.3822 [nucl-th].

[29] E. Epelbaum, W. Gloeckle and Ulf-G. Meissner. Eur. Rhys. J. A19: 125-137, (2004).

[30] C.-J.Yang, Ch.Elster and D.R.Phillips Phys. Rev. C 77, 014002 (2008).

[31] C.-J.Yang, Ch.Elster and D.R.Phillips Phys. Rev. C 80, 034002 (2009).

[32] C. J. Yang, C. Elster and D. R. Phillips, arXiv:0905.4943 [nucl-th].

[33] H.-W. Hammer and T. Mehen, Nucl. Phys. A 690, 535 (2001).

[34] I. R. Afnan and D. R. Phillips, Phys. Rev. C 69, 034010 (2004).

[35] M. Pavon Valderrama, E. Ruiz Arriola, nucl-th/0407113v1.

[36] see http://nn-online.org/

[37] S. R. Beane, P. F. Bedaque, L. Childress, A. Kryjevski, J. McGuire and U. v. Kolck, Phys. Rev. A 64, 042103 (2001).

[38] T. Frederico, V. S. Timoteo and L. Tomio, Nucl. Phys. A 653, 209 (1999).

[39] V. S. Timoteo, T. Frederico, A. Delfino and L. Tomio, Phys. Lett. B 621, 109 (2005).

[40] R. Machleidt, Phys. Rev. C63, 024001 (2001). 